\title{
Forgetting in Creative Problem Solving for College Students
}

\author{
Yue Zhao ${ }^{1, *}$ \\ ${ }^{1}$ Faculty of Education, Beijing Normal University, Beijing, 100875, China \\ ${ }^{*}$ Corresponding author email: 201811010189@mail.bnu.edu.cn
}

\begin{abstract}
As more and more benefits of forgetting have been found in recent studies, whether forgetting could promote individual's ability of creative problem solving remains a controversial debate. Once the associations between forgetting and creative problem solving are proved, we could promote one's ability of creative problem solving by inducing specific forgetting. This paper discusses the effect of two types of forgetting: a) the retrieval-induced forgetting and b) the forgetting during incubation, in benefiting creative problem solving by introducing and analysing the relevant experiments. The results reveal that retrieval-induced forgetting only works when previous mental fixations occurs and the promotion varies when solving different types of problems. The level of RIF is irrelevant to the performance in solving closed-ended creative problems and high level of RIF even impairs the creativity when solving open-ended problems. Forgetting during incubation cannot explain the incubation effect. The spreading activation of relevant information or the unconscious work are more likely to be the possible reasons. In conclusion, this paper brings about the discussions about the work conditions and effects of forgetting in creative problem solving.
\end{abstract}

Keywords: Retrieval-induced forgetting, incubation, creative problem solving

\section{INTRODUCTION}

Forgetting is a natural and common process for all human beings and it refers to "the apparent loss or modification of information already encoded and stored in an individual's short or long-term memory" [1].

The study of forgetting has a history for over 100 years. In 1885, Herman Ebbinghaus was the first psychologist to study forgetting by a six-year quantitative research and concluded the famous Ebbinghaus forgetting curve, which indicated the phenomenon and rules of memory decay [2]. Then many psychologists started to explore the possible causes of forgetting. At first, psychologists attribute forgetting to the interference of other information. The interference theory claims that the memory may be interfered by previous information (proactive) or new information (retroactive) because of the competition in memory sources and become distorted or disrupted [3]. In 1958 and 1959, Brown [4], Peterson and Peterson [5] raised another explanation, decay trace theory. They assumed that memory left a trace in the brain and when the trace decayed, the memory was gone. This theory regards memory as a result of the passage of time and has been supported by recent advances in neuroscience [6]. In 1974, Tulving put forward the theory that our memory was cue-dependent, which meant our long-term memory always existed in our brain but without proper cues we could not retrieve them successfully [7]. In 2003, interference theory was criticized by Anderson, the supporter of inhibition theory who emphasized that "it was the executive control mechanism that overcomes interference - inhibition - that causes us to forget, not the competition itself' [8]. Lastly, there are also some organic causes of forgetting related to physiological or brain damage such as Alzheimer's, amnesia and dementia. In conclusion, the possible causes of forgetting contain decay, interference, cues loss, inhibition and organic causes.

Besides the cause of forgetting, the advantages and disadvantages of forgetting are the main research topics of psychologists as well. On the negative side, forgetting could undermine our good intentions by disrupting our sympathetic preference for the unlucky group [9]. On the positive side, forgetting has many advantages: facilitating remembering, promoting second language acquisition, helping us imagine the future more positively and promoting creative problem solving. Firstly, for facilitating remembering, Anderson proved that forgetting facilitated remembering words by overcoming interference during retrieval [10]. Except for remembering words, forgetting is also helpful in 
remembering meaningful stimuli [11] and arithmetic facts [12]. Secondly, for second language learning, an experiment has proved that forgetting inhibited the phonology of native language to promote individuals' second language acquisition [13]. Thirdly, forgetting is associated with increased positivity when imagining the future. Individuals who exhibit higher levels of retrieval-induced forgetting imagine fewer negative episodic future events than individuals who exhibit lower levels of retrieval-induced forgetting [14].

However, whether forgetting can benefit creative problem solving is still a controversial debate. On the one hand, some researches support that forgetting can promote creative problem solving. Storm and Angello connected forgetting to creative problem solving and explored the relationship of these two processes in 2010 . They testified that retrieval-induced forgetting made contribution to creative problem solving by reducing the interference of mental fixation [15]. This kind of forgetting is cue-dependent [16]. On the other hand, the level of retrieval-induced forgetting is proved to negatively predict the performance in solving some kinds of creative problems [17]. In addition, the memory decay during incubation period is also proved to be relevant to the creative problem solving in recent researches $[18,19]$.

According to the above literature, the mechanisms and necessary conditions of forgetting affecting creative problem solving remains unclear. In the existing research, college students are chosen as the target subjects to explore the relationship between forgetting and creative problem solving because they were available. In addition, promoting creative problem-solving ability is of great importance for these students who are about to face the fierce competition in the modern society. As a result, this paper will clarify the role of forgetting in creative problem solving and summarize in what conditions forgetting matters and how it matters for college students.

\section{FORGETTING IN CREATIVE PROBLEM SOLVING}

\subsection{Retrieval-induced forgetting in creative problem solving}

In order to explore the role of retrieval-induced forgetting in creative problem solving, we need to clarify the concepts and measure the levels of retrieval-induced forgetting and the ability of creative problem solving.

Retrieval-induced forgetting (RIF) is a kind of forgetting happens when individuals try to retrieve something in their mind [10]. The successful retrieval of the target information can lead to the inhibition of nontarget information thus the nontarget information becomes vulnerable to forget. Anderson designed a retrieval-induced forgetting paradigm to testify this kind of forgetting and this paradigm was used as the measurement of RIF level in many following research. At the first stage of this experiment, participants were exposed to a series of category-exemplar pairs such as 'fruit-orange' and 'tree-redwood' and were asked to memorize the pairs. At the second stage, participants took practices to retrieve the exemplars paired with categories with the cues of first two initials. Only half of the pairs presented at the first stage were selected to practice. Finally, a test was taken by participants to recall the proper category exemplars with the cues of the first initials. The phenomenon of RIF was observed when the recall performance for nonpracticed exemplars from nonpracticed categories was better than the recall performance for nonpracticed exemplars from practiced categories, which meant the memory of nonpracticed exemplars from practiced categories was inhibited by the prior practice. By comparing recall performance for nonpracticed exemplars from practiced categories with recall performance for nonpracticed exemplars from nonpracticed categories, we can conclude one's level of RIF.

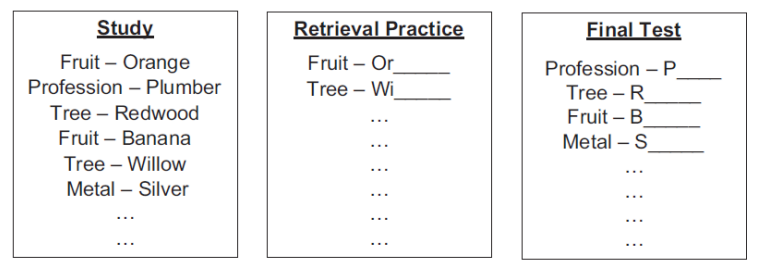

Figure 1 Schematic of the standard retrieval-practice paradigm [20]

Creative problem solving is a proven method for approaching a problem or a challenge in an imaginative and innovative way [21]. In the psychological experiments, Remote Associate Test [22] is usually used to evaluate one' s ability of creative problem solving. In the Remote Associate Test, participants are required to connect three cue words seemingly irrelevant with one target word, which is associated with all the three words in semantic way. For instance, 'manners' 'tennis' and 'round' are the three cues and the correct target associate can be 'table'. The RAT problems are difficult to solve but the number of RAT problems solved in a limited time can indicate one's ability of creative problem solving. As a result, RAT is used as a classical test of creativity.

When it comes to the association between retrieval-induced forgetting and creative problem solving, we need to introduce another variable-mental fixation. Retrieval-induced forgetting could inhibit mental fixation, which is able to hinder the process of creative problem solving. Exposing the participants to the intrusive associates of cue words before RAT impairs their performance in creative problem solving 
[23]. While Storm and Angello [15] found that individuals with high level of RIF could effectively overcome the interference of mental fixation and perform better in the RAT. In the experiment, participants were measured the level of RIF by RIF paradigm at first, and then were presented some unappropriated pairs of cue words before the final Remote Associate Test. With the time engaged in the RAT increasing, the fixation effect (subtracting the percentage of problems solved correctly by participants in the fixation condition from that of participants in the baseline condition) accumulated all the time. This result revealed that individuals who demonstrated higher level of RIF were more competent in creative problem solving under fixation.

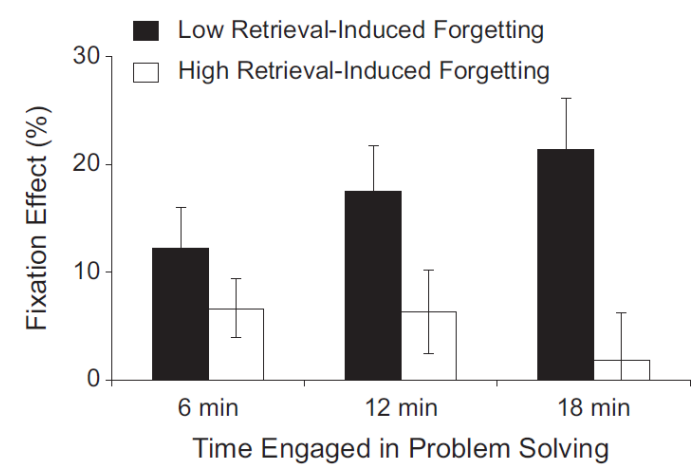

Figure 2 Cumulative fixation effects as a function of problem-solving block for participants exhibiting high and low levels of retrieval-induced forgetting [24]

However, high level of RIF does not promote creative problem solving all the time. The performance superiority of high-RIF participants disappears when there is no fixation prior to RAT. In the baseline condition, individuals who demonstrate a lower level of RIF perform better! In this situation, high level of RIF may harm one's creativity by forgetting the possible remote associates. Only when the benefits of inhibiting mental fixation outweighs the benefits of forgetting possible solutions, can the retrieval-induced forgetting promote the performance of creative problem solving. That's why the situations in fixation and baseline condition are so different [25].

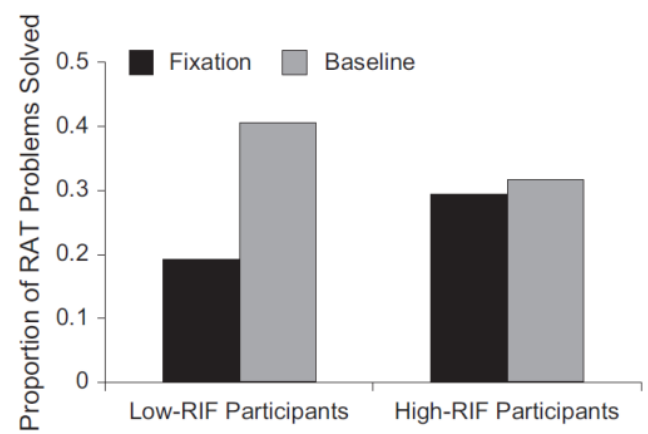

Figure 3 RAT problem-solving performance observed by Storm and Angello [20]
The type of creative problem also affects the retrieval-induced forgetting effect in creative problem solving. Wei and Yunn [17] conducted two experiments to explore the role of RIF in open-ended and closed-ended creative problem solving. Open-ended creative problem refers to those problems which had divergent correct answers. In this experiment, researchers used the Chinese version of the Creative Thinking Test [26] as the open-ended creative problem to measure one's divergent creative thinking. The evaluation of the answer's quality usually lies in three aspects: fluency, flexibility and originality. The fluency could be reflected in the number of answers generated in a limited time. The flexibility equals the number of categories. The originality equals in the answers that were not common and seldom generated. While the closed-ended creative problem equals those problem with specific and limited correct answers. RAT was a typical kind of closed-ended creative problem and in this experiment, 2-4-6 problem [27], another classical closed-ended creative problem, was used as the measurement tool. In the 2-4-6 problem task, participants were asked to discover the predetermined rules of the number triples. They had 12 opportunities to attempt. The new-perspective hypothesis and the correctness of problem solving were the main indices to indicate one's closed-ended problem solving ability. A Chinese version of RIF paradigm was selected to evaluate the participants' level of RIF. All the participants took the three tests. It was observed that low-RIF participants performed better at open-ended creative problem-solving task while the performance on the closed-ended problem test was not correlated to the level of RIF.

In conclusion, retrieval-induced forgetting can facilitate or impair the ability of creative problem solving in different situations. It is more likely to benefit creative problem solving when individuals are faced with the interference of other intrusive responses or nontarget information. This kind of forgetting can play an important role in overcoming existing mental fixation. While in other situation, especially for solving the open-ended problems, the retrieval-induced forgetting may impair the ability of creative problem solving by making the possible solutions in their mind less available.

\subsection{Forgetting during incubation in creative problem solving}

Many empirical researches have proved that incubation could improve individual's performance in creative problem solving. The 'incubation' refers to 'superior performance for those subjects who return to the problem after a delay rather than working continuously on the problem' [28]. The phenomenon that incubation brings about creativity improvement is 
called 'incubation effect', which means after individual was temporarily distracted from the unsolved problem, he/she could come up with some unexpected new solutions. The psychologists raise many hypotheses to explain the incubation effect: spreading activation[29], selective forgetting[30], intermittent conscious work, beneficial forgetting, unconscious work[18] and forgetting-fixation[28]. According to the existing hypotheses, we can find out that forgetting is a critical hypothesis for how incubation effect functions. Many experiments have been conducted to test these hypotheses and they generated opposite conclusions.

Smith and Blankenship conducted three relevant experiments to testify forgetting-fixation hypothesis. In the first experiment, researchers presented participants 15 rebuses with useful clues, followed by a rebus with misleading clues, which was designed to induce participants' mental fixation to use the intrusive clues to solve the 16th rebus. Then after an interval ( $0 \mathrm{~min}, 5$ min and $15 \mathrm{~min}$ ) filled or unfilled with a demanding 'music perception task', the subjects were asked to solve the 16th rebus first and then recall its associated clues. The improvements in creative problem solving was at an average of $30 \%$ after incubation. The control group (without interval) not only showed no evident improvements but also recalled the misleading clues faster, which supported the forgetting-fixation hypothesis. In experiment 2 , the subjects were first presented a rebus with misleading clues and then went through an interval, $5 \mathrm{~min}$ or $15 \mathrm{~min}$, filled or unfilled with other tasks. After the incubation, they were required to resolve the same rebus with useful clues and recall the former clue. This time, incubation effect was only observed in the 15-min group as more forgetting and more solutions were found after a longer interval. In addition, there was no obvious evidence showing that filler tasks promoted the incubation effect. In experiment 3, researchers filled different types of activities into the incubation period for the incubation group and controlled other factors. The results showed that the type of activities did not have an influence on the incubation effect. Forgetting was also observed in this experiment. In summary, forgetting during incubation period had been proved to correlate to the improvements of creative problem solving while continued work on unsolved problems during incubation (without filled tasks) appeared to be helpless [28].

However, there were also some experiments' results that didn't support forgetting during incubation could benefits creative problem solving. Ut $\mathrm{Na} \mathrm{Sio}$ and Elisabeth Rudowicz tested the effect of selective forgetting in 2007. They recruited 57 college students or recent graduates who was either a GO expert or a GO novice and involved them in solving Remote Associated Test (RAT) problems and lexical decision tasks (LDT). The RAT problems were divided into 3 types, including neutral (the three words and solutions were unrelated to the GO), GO-relevant (all the words and solutions were GO-related) and GO-misleading (the first two words were GO-related and the third words and solutions were GO-unrelated). The LDT was about distinguishing real words from pseudo-words to measure the subjects' sensibility to the words presented in the RAT. At first, all the participants were presented 6 neutral RAT problems, 6 GO-relevant RAT problems and 6 GO-misleading RAT problems. In the Immediate Condition, subjects continued to do the LDT right after the RAT test. In the Rest Condition, subjects took a 2-min rest with soft music and then did the LDT. In the incubation condition, the 2-min interval was filled with other tasks. Finally, in order to measure their improvements in creative problem solving, all the subjects were retested on the RAT test. Researchers analysed the participants' performance in RAT and LDT tests and found that GO-experts under the incubation condition were not less sensitive to irrelevant concepts (intrusive GO-related words of GO-misleading RAT problems), which meant that they did not selectively forgot some irrelevant information during incubation. On the contrary, both experts and novices showed more familiarity with the relevant concepts of the unsolved RAT, which revealed that it was the spreading activation hypothesis not the selective forgetting hypothesis explain the incubation effect [31].

Further research rejects the effect of forgetting during incubation. The similarity of incubation activity and target task, which is supposed to induce more interference-based forgetting of information in target task, is unable to promote participants' performances in target task [32]. Incubation doesn't influence the number of fixation or individual's sensibility to the fixation words, neither [19].

In conclusion, the initial researches prove that forgetting happens during incubation period. However, recent researches explore the underlying mechanisms of incubation effect and reject the forgetting hypothesis. The spreading activation of relevant information or the unconscious work are the possible undermining mechanisms of incubation period affecting the creative problem solving.

\section{CONCLUSION}

Forgetting is a natural part of cognitive process which has advantages and disadvantages at the same time. On the positive side, forgetting can facilitate remembering, promote second language learning, increase the positivity of one's imagination of future and so on. On the negative side, forgetting can cause many troubles in our daily life and undermine our good intentions by forgetting what happened to the unlucky ones. However, among all the merits and drawbacks, whether forgetting could benefit creative problem solving is still controversial and unclear. If we can 
figure out the associations between forgetting and creative problem solving, we may improve one's ability of creative problem solving by intervening his/her process of forgetting, for example, inducing specific forgetting. Many researchers chose college students as subjects to study this topic. They conducted various experiments but came to different conclusions. This paper mainly discusses about the work conditions and undermining mechanisms of two types of forgetting in creative problem solving.

The first type of forgetting is retrieval-induced forgetting. We introduce two experiments to testify whether RIF can improve the ability of creative problem solving and other two experiments to explore the associations between the RIF and different types (open-ended or closed-ended) of creative problem solving. After analysing the results of all the experiments, we conclude that RIF only works when mental fixations occurs. When solving open-ended creative problems, RIF harms one' $\mathrm{s}$ creativity by making the potential solutions less recallable. When solving closed-ended creative problems, RIF does not correlate with the ability of creative problem solving. The second type of forgetting is forgetting during incubation period because forgetting is one of important hypotheses for the incubation effect. We introduce 3 experiments to test the forgetting-fixation hypothesis and another one experiment to test selective forgetting hypothesis. The results indicate that the forgetting during incubation indeed exist but is not the underlying mechanism of the incubation effect. As a result, forgetting hypotheses during incubation cannot explain the promotion of creative problem solving.

At last, there are still some limitations in this paper. We only focus on two main types of forgetting which have been proved to be relevant to the creative forgetting solving and the number of experiments we introduced is limited. The historical development of research about forgetting in creative problem solving has not been clarified, neither. Further researches are encouraged to explore the effect of more types of forgetting in creative problem solving and to clarify and analyse the history of this research area.

\section{REFERENCES}

[1] Wikipedia contributors. (2021, May 23). Forgetting. In Wikipedia, The Free Encyclopedia. Retrieved 06:35, September 5, 2021, from https://en.wikipedia.org/w/index.php?title=Fo rgetting\&oldid $=1024607612$

[2] G. S. Robertson (2009). A Brief History of the Mathematical Definition of Forgetting Curves. Friday. June, 26.

[3] B. J. Underwood (1957). Interference and forgetting. Psychological review, 64(1), 49.
[4] Brown, John (1958). Some Tests of the Decay Theory of Immediate Memory. Quarterly Journal of Experimental Psychology, 10, 12-21.

[5] L. Peterson, \& M. J. Peterson (1959). Short-term retention of individual verbal items. Journal of experimental psychology, 58(3), 193.

[6] O. Hardt, K. Nader, \& L. Nadel (2013). Decay happens: the role of active forgetting in memory. Trends in cognitive sciences, 17(3), $111-120$

[7] E. Tulving (1974). Cue-dependent forgetting: When we forget something we once knew, it does not necessarily mean that the memory trace has been lost; it may only be inaccessible. American Scientist, 62(1), 74-82.

[8] M. C. Anderson (2003). Rethinking interference theory: Executive control and the mechanisms of forgetting. Journal of memory and language, 49(4), $415-445$

[9] K. R. Olson, A. S. Heberlein , E. Kensinger, C. Burrows, C. S. Dweck, E. S. Spelke, \& M. R. Banaji (2013). The role of forgetting in undermining good intentions. PloS one, 8(11), e79091.

[10] M. C. Anderson, R. A. Bjork , \& E. L. Bjork, (1994). Remembering can cause forgetting: retrieval dynamics in long-term memory. Journal of Experimental Psychology: Learning, Memory, and Cognition, 20(5), 1063.

[11] M. MacLeod (2002). Retrieval-induced forgetting in eyewitness memory: Forgetting as a consequence of remembering. Applied Cognitive Psychology: The Official Journal of the Society for Applied Research in Memory and Cognition, 16(2), 135-149.

[12] J. I.Campbell, \& V. A. Thompson (2012). Retrieval-induced forgetting of arithmetic facts. Journal of Experimental Psychology: Learning, Memory, and Cognition, 38(1), 118.

[13] B. J. Levy, N. D. McVeigh, A. Marful, \& M. C. Anderson (2007). Inhibiting your native language: The role of retrieval-induced forgetting during second-language acquisition. Psychological Science, 18(1), 29-34.

[14] S. Giebl, B. C. Storm, D. R. Buchli, E. L. Bjork, \& R. A. Bjork (2016). Retrieval-induced forgetting is associated with increased positivity when imagining the future. Quarterly Journal of Experimental Psychology, 69(2), 351-360.

[15] B. C. Storm, G. Angello \& E. L. Bjork (2011). 
Thinking can cause forgetting: Memory dynamics in creative problem solving. Journal of Experimental Psychology: Learning, Memory, and Cognition, 37(5), 1287.

[16] B. C., Storm \& R. H., Koppel (2012). Testing the cue dependence of problem-solving-induced forgetting. The Journal of Problem Solving, 4(2), 4.

[17] W. L., Lin \& Y. W., Lien (2013). Exploration of the relationships between retrieval-induced forgetting effects with open-ended versus closed-ended creative problem solving. Thinking skills and creativity, 10, 40-49.

[18] K. J., Gilhooly(2016). Incubation and intuition in creative problem solving. Frontiers in psychology, 7, 1076.

[19] E.,VALUEVA \& N., LAPTEVA (2020). Do We Need to Forget Fixations to Incubate? A Paradox of the Forgetting Fixation Theory. Psychology, 17(4), 682-695.

[20] B. C. Storm(2011). The benefit of forgetting in thinking and remembering. Current Directions in Psychological Science, 20(5), 291-295.

[21] Wikipedia contributors. (2021, March 10). Creative problem-solving. In Wikipedia, The Free Encyclopedia. Retrieved 03:20, September 23, 2021,

from https://en.wikipedia.org/w/index.php?title $=\mathrm{Cr}$ eative problem-solving\&oldid $=1011450550$

[22] S., Mednick (1962). The associative basis of the creative process. Psychological review, 69(3), 220.

[23] S.M.,Smith \& S.E. Blankenship (1991). Incubation and the persistence of fixation in problem solving. American Journal of Psychology, 104, 61-87.

[24] B. C., Storm, \& G. Angello (2010). Overcoming fixation: Creative problem solving and retrieval-induced forgetting. Psychological Science, 21(9), 1263-1265.

[25] A.S., Bristol \& I.V., Viskontas (2006). Dynamic processes within associative memory stores: Piecing together the neural basis of creative cognition. In J.C. Kaufman \& J. Baer (Eds.), Creativity and reason in cognitive development (pp. 60-80). New York: Cambridge University Press.

[26] C.Wu, -C.(1998). The Chinese version of the creative thinking test. Taiwan: Foundation for Scholarly Exchange.

[27] P. C., Wason (1960). On the failure to eliminate hypotheses in a conceptual task. Quarterly journal of experimental psychology, 12(3), 129-140.

[28] S. M. Smith \& S.E.Blankenship (1989). Incubation effects. Bulletin of the Psychonomic Society, 27(4), 311-314.

[29] S. M. Smith(1995). Fixation, incubation, and insight in memory and creative thinking. The creative cognition approach, 135, 156.

[30] I., Yaniv \& D. E. Meyer (1987). Activation and metacognition of inaccessible stored information: potential bases for incubation effects in problem solving. Journal of Experimental Psychology: Learning, Memory, and Cognition, 13(2), 187.

[31] U. N. Sio \& E. Rudowicz, (2007). The role of an incubation period in creative problem solving. Creativity Research Journal,19(2-3), 307-318.

[32] K. J. Gilhooly, G.,Georgiou, \& U. Devery, (2013). Incubation and creativity: Do something different. Thinking \& Reasoning, 19(2), 137-149. 